\title{
Effect of peat humic acid on the composition of cytosolic soluble carbohydrates and lipids in Alternaria alternata
}

\author{
Fedoseeva $\mathrm{E}^{1}$, Tereshina V. ${ }^{2}$, Danilova O. ${ }^{2}$, Prudnikova E. ${ }^{3}$, Terekhova V. ${ }^{3}$ \\ ${ }^{1}$ Pirogov Russian National Research Medical University, Moscow, Russia, \\ elenfedoseeva@gmail.com \\ ${ }^{2}$ Winogradsky Institute of Microbiology, Research Center of Biotechnology of the Russian \\ Academy of Sciences, Moscow, Russia \\ ${ }^{3}$ Lomonosov Moscow State University, Moscow, Russia
}

Keywords: humic acid, micromycetes, Alternaria, stress, osmolytes, lipids, sterols, trehalose

doi: 10.36291/HIT.2019.fedoseeva.070

Humic substances can influence on various plant and microbial cell components such as plasma membrane, mitochondria, DNA containing components, and cytosol [3]. Such changes can be considered both positive and stressful for organisms. It has been known that fungi can respond to stress changing the composition of membrane lipids and synthesizing protective osmolytes [1] Melanin-synthesizing fungi, as a rule, are characterized by high resistance to external influences. The aim was to study the effect of humic acid (HA) on cytosolic soluble carbohydrates and lipids in melanin-synthesizing fungus Alternaria alternata to understand whether the action of $\mathrm{HA}$ is stressful.

Fungal culture was cultivated on a liquid Chapek medium with the addition of peat HA (100-200 mg/L) and without it as a control. Mycelium was investigated in trophophase. The method of lipid extraction inhibiting phospholipases was used to analyze the lipid composition of fungal cells. Quantitative analysis of the membrane lipids was performed using two-dimensional thin-layer chromatography. The method of gas-liquid chromatography (GLC) was used to study the composition of fatty acids in fractions of polar or individual lipids. The osmolytes were determined by the method of GLC trimethylsilyl sugar derivatives with an internal standard after extraction and purification.

Mannitol and trehalose dominated within the composition of osmolytes. Under the action of peat HA level of mannitol reduced, while the level of trehalose increased (by 1.52 times). Within membrane lipids, phosphatidylcholines, phosphatidylethanolamines, and phosphatidic acids dominated. However, more distinct effect of HA was observed in the structure of other lipid groups, namely an increase in the proportion of sterols and sphingolipids. It has been known that an increase in trehalose is a response to heat shock, in sterols - to a microbial attack; and a high proportion of phosphatidic acids in the membranes can indicate an overall adverse effect on living organisms [1, 2]. Thus the increase in the proportions of sterols and trehalose in our experimental research can be considered as stress response of fungal cells on peat HA.

The study was supported by the Russian Foundation for Basic Research (project 1804-01218).

\section{References}

1. Ianutsevich E.A. et al. // Microbiology (SGM). 2016. 162(6):989-99.

2. Mantil E. et al. // Colloids and Surfaces B: Biointerfaces. 2019. 178:94-102.

3. Zandonadi D.B. et al. // Planta. 2007. 225: 1583-1595. 